\title{
XÂY DỬNG WEBSITE GIAO THÔNG XE BUS HÀ NỘI
}

\author{
PGS. TS. NGUYẼ̃N TRƯƠ'NG XUÂN(1), \\ KS. NGUYẼ̃N TRƯỜNG LINH(1), KS. MAI VĂN SỸ ${ }^{(2)}$ \\ (1) Trường Đại học Mỏ-Địa chất \\ (2) Công ty Cổ phần Tư vấn thiết kế công trình xây dựng Hải Phòng
}

\section{Tóm tắt:}

Bài báo giới thiệu Website giao thông xe bus trên địa bàn Thành phố Hà Nội được thiết kế và phát triển trên cơ sở ứng dụng ngôn ngữ lập trình JavaScript và công nghệ Map API nhằm hỗ trợ tra cứu thông tin giao thông xe bus trên máy tính cá nhân hoặc điện thoại di động có kết nối Internet.

\section{Mở đầu}

Giao thông luôn là một vấn đề hết sức phức tạp, nhất là đối với thủ đô Hà Nội đang trong giai đoạn phát triển hiện nay. Hà Nội thay đổi liên lục về kiến trúc cơ sở hạ tầng, các con đường, các khu dân cư, các khu công nghiệp mới mọc lên nhanh chóng, khiến cho người tham gia giao thông gặp nhiều khó khăn. Thêm vào đó là xu hướng sử dụng các phương tiện giao thông công cộng của người dân, nhất là xe bus, phương tiện bắt đầu phổ biến ở Việt Nam. Để giúp mọi người có thể nhanh chóng thu nhận các thông tin cần thiết khi tham gia giao thông công cộng ở Hà Nội, Website giao thông xe bus Hà Nội sẽ bước đầu giúp tra cứu các thông tin về các con đường, các địa điểm, khu vực và các tuyến xe bus một cách nhanh chóng trên máy tính cá nhân hay điện thoại di động có kết nối Internet.

\section{Công nghệ}

Để thực hiện ý tưởng này, ta sử dụng ngôn ngữ lập trình JavaScript và Map API.

\section{Xây dụng Website}

\subsection{Cấu trúc Website}

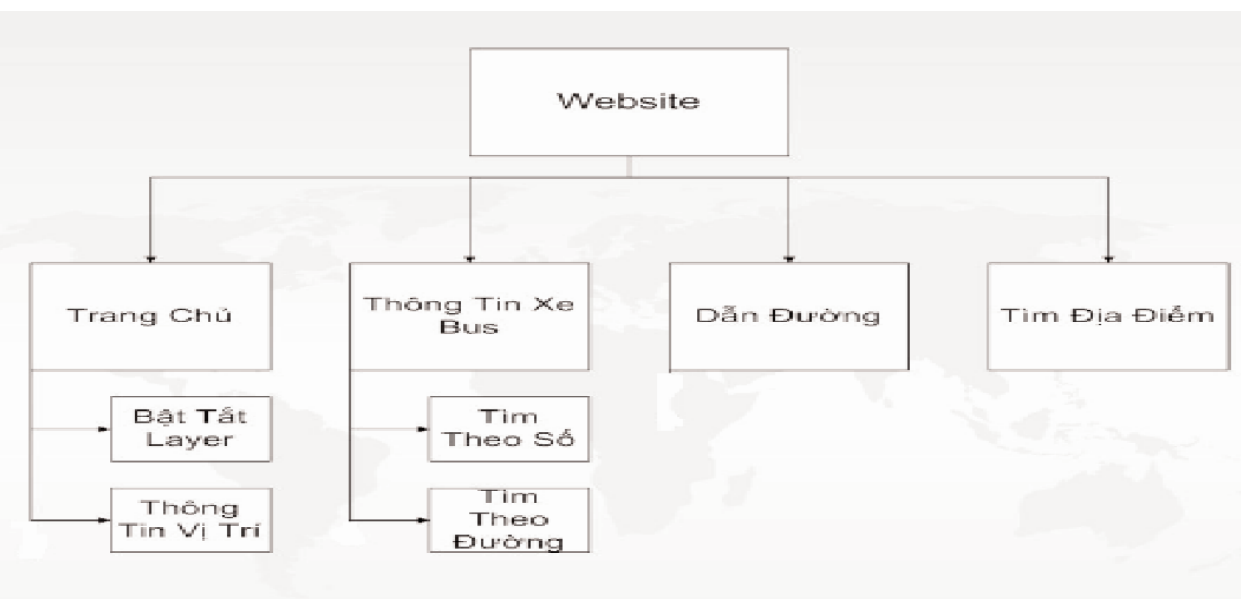

Hình 1: Sơ đồ cấu trúc Website 


\subsubsection{Trang chủ}

Trang chủ là phần giao diện chính của chương trình khi hiển thị, phần này có chức năng hiển thị bản đồ phần lớn màn hình, có các phần bật tắt Layer để xem các thông tin về bến xe khách, bến xe bus, bến xe taxi, ...

\subsubsection{Thông tin xe bus}

Thông tin xe bus là mục cho người dùng tra cứu thông tin xe bus theo sô hiệu, theo tuyến hoặc theo đường. Mục này có phần hiển thị bản đồ có tuyến xe bus, tuyến đường, và phần thông tin lộ trình, giờ bắt đầu, kết thúc, giá vé...

\subsubsection{Dẫn đường}

Dẫn đường là mục người dùng có thể tìm đường đi ngắn nhất từ hai vị trí cho trước. Mục này có phần hiển thị bản đồ có vẽ đường đi nối hai địa điểm đã nhập và phần chỉ dẫn cụ thể cách đi từ địa điểm này đến địa điểm kia rất chi tiết. Khi click vào vị trí nào trên hướng dẫn bản đồ sẽ hiển thị thông tin vị trí đó trên bản đồ một cách trực quan.

\subsubsection{Tìm địa điểm}

Tìm địa điểm là mục người dùng có thể tìm địa điểm muốn đến trên bản đồ khi nhập tên điểm đó vào chương trình. Mục này có phần hiển thị bản đồ có vị trí điểm đó bằng việc đánh dấu đỏ trên bản đồ, khi muốn tìm hiểu kĩ người dùng zoom to vị trí đó và

biết đường nó nằm trên đường nào, quận nào, từ đó có những thông tin hữu ích nhất.

\subsection{Xây dựng cơ sở dũ liệu}

Cơ sở dữ liệu của website được xây dựng bằng phần mềm My SQL server. Dữ liệu chính là bản đồ, website sử dụng bản đồ nền của Google, ngoài ra còn các bảng dữ liệu khác được mô tả dưới đây.

ThuocTinhXe (MaXeBus, SoCuaTuyenXe, LoTrinh, GiaVe, GioKetThuc, GioXuatPhat)

\begin{tabular}{|c|c|c|c|}
\hline Field Name & Data Type & Field Size & Not Null \\
\hline MaXeBus & INTEGER & 11 & True \\
\hline SoCuaTuyenXe & INTEGER & 11 & True \\
\hline LoTrinh & VARCHAR & 40 & True \\
\hline GiaVe & FLOAT & 9 & True \\
\hline GioKetThuc & TIME & 0 & True \\
\hline GioXuatPhat & TIME & 0 & True \\
\hline
\end{tabular}

Bảng 1: Bảng ThuocTinhXe 
TuyenDuong_Xe (MaDuong_Xe, MaDuong, TenDuong)

\begin{tabular}{|c|c|c|c|}
\hline Field Name & Data Type & Field Size & Not Null \\
\hline MaDuong_Xe & INTEGER & 11 & True \\
\hline MaDuong & INTEGER & 11 & True \\
\hline TenDuong & VARCHAR & 60 & True \\
\hline
\end{tabular}

Bảng 2: Bảng TuyenDuong_Xe

\subsection{Xây dựng các chức năng của chương trình}

\subsection{1. Đăng ký Code của Google Maps API}

Trong phần này sẽ giới thiệu cách lấy key của Google Maps Api. Chỉ khi có Key thì mới có thể tạo được các ứng dụng của Google Maps. Đầu tiên phải đăng nhập vào tài khoản Google. Sau đó vào trang web sau: http://www.google.com/apis/maps/signup.html để đăng ký nhận Google API key. Nếu không đăng nhập vào thì khi ấn Generate API Key thì trang web sẽ tự động chuyển tới trang đăng nhập vào tài khoản Google và yêu cầu đăng nhập vào. Nên nhớ đánh tên website vào ô My web site URL.

Sau khi đăng nhập vào và bấm vào nút Generate API Key thì chúng ta sẽ nhận được key. Key có dạng như sau: BQIAAAAuPsJpk3MBtDpJ4G8cqBnjRRaG TYH6UMI8mADNa0YKuWNNa8VNxQCzVBXTx2DYyXGsTOxpWhvIG7Djw

Bây giờ ta sẽ tìm hiểu một chút về cách sử dụng key API này. Chúng ta sẽ tạo một trang web đơn giản có nhúng key API vào.

$<\mathrm{html}>$

$<$ head $>$

<title>Google Maps JavaScript API Example</title>

<script src="http://maps.google.com/maps?file=api\&amp;v=2\&amp;key= ABQIAAAAuPsJpk3MBtDpJ4G8cqBnjRRaGTYH6UMI8mADNaOYKuWNNa8VNxQCzVBX Tx2DYyXGsTOxpWhvIG7Djw" type="text/javascript"></script>

<script type="text/javascript">

function load () \{

if (GBrowserlsCompatible ()) \{

var map = new GMap2 (document.getElementByld ("map"));

map.setCenter (new GLatLng (21.0430806705295, 105.77565103769), 13);

\}

\}$<$ script $>$ 


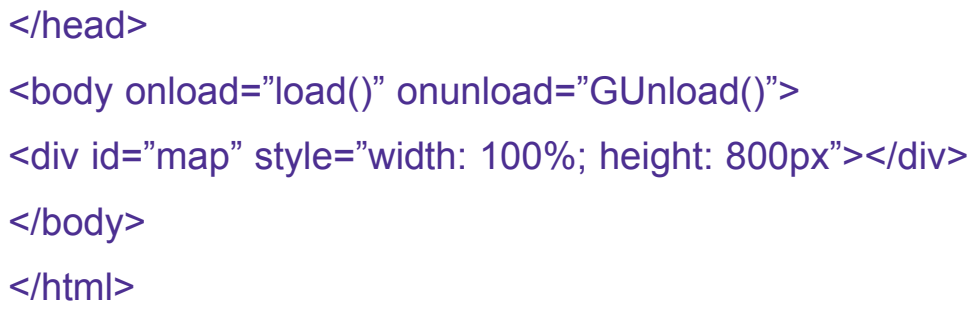

\subsubsection{Lập trình các chức năng hiển thị của website}

Sau khi tạo được một web đơn giản có hiển thị được bản đồ của Google Maps bây giờ chúng ta bắt đầu đi sâu tìm hiểu các hàm sẵn có mà Google Maps Api đã cung cấp cho ta để từ đó có thể xây dựng nên một website theo mục đích. Có thể vào địa chỉ sau để tìm hiểu thêm http://code.google.com/apis/maps/. Trong website có nhiều lựa chọn và đồ án này được thực hiện bằng Google Maps Javascript $\mathrm{API}$.

Đặc thù website sẽ hiển thị bản đồ số để cho người dùng dễ dàng quan sát, trên đó người dùng có thể zoom tới các địa điểm mình muốn bằng cách lăn chuột giữa hay click vào các biểu tượng ở trên bản đồ. Ngoài ra chức năng chính của website là tìm kiếm thông tin về các tuyến xe bus theo tên xe, theo tuyến đường, tìm kiếm địa điểm, dẫn đường đi giữa hai địa điểm và chức năng thêm địa điểm lên bản đồ cho riêng mình. Để hiểu hơn về các chức năng chúng ta sẽ đi sâu nghiên cứu về các chức năng này

a. Thiết kế giao diện chương trình

Giao diện chương trình được thực hiện thuần code html gồm các phần chính là banner, menu, phần thiết bật tắt Layer và phần hiển thị bản đồ.

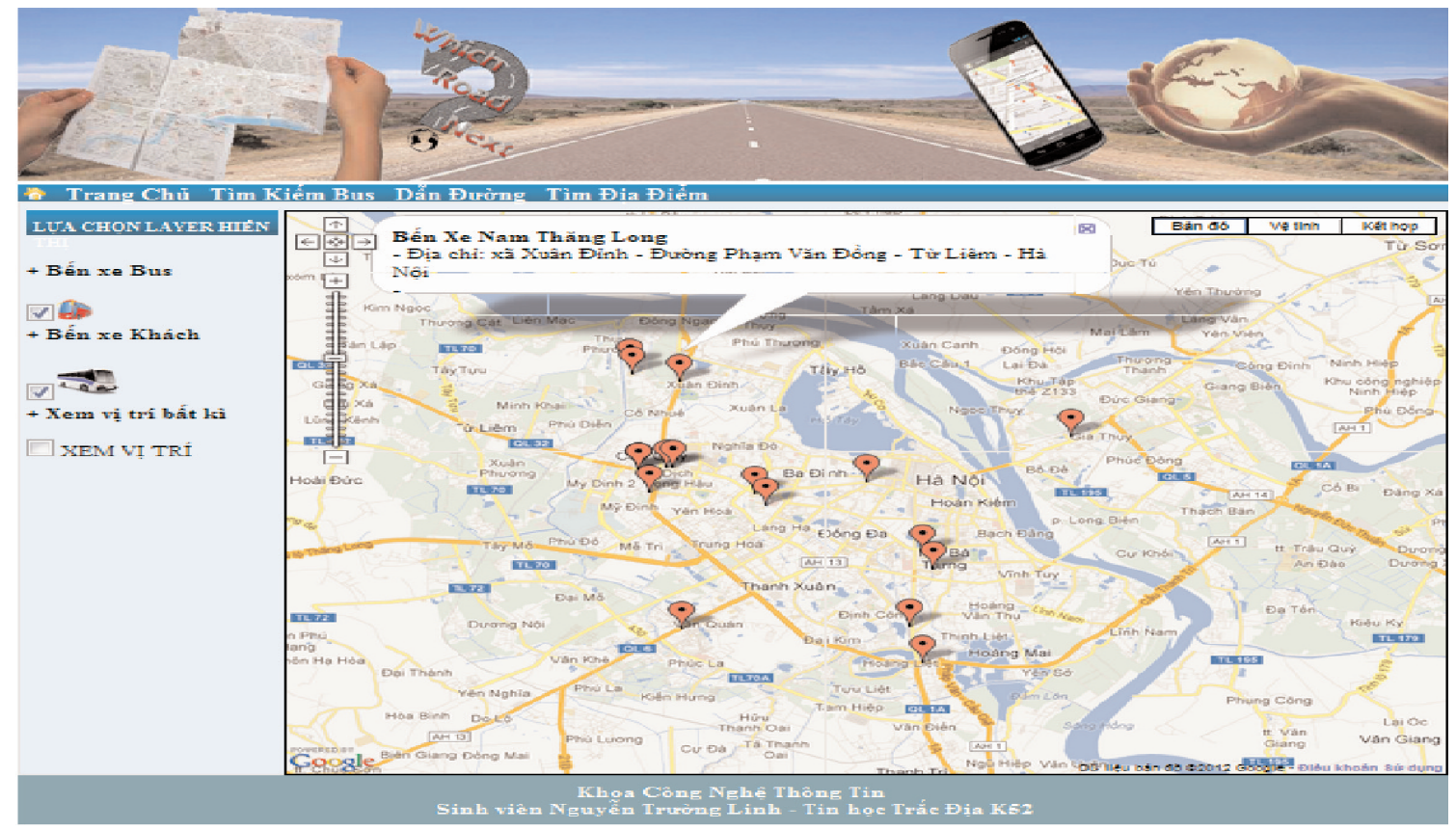

Hình 2: Giao diện chương trình 
b. Thực hiện chức năng tìm kiếm xe bus theo số xe

Chức năng giúp người dùng biết được các thông tin cần tìm kiếm về tuyến xe, để từ đó có những quyết định đúng đắn khi tham gia giao thông.

Sau khi nhập số xe cần tìm kiếm vào ô trên rồi click vào nút Tìm số thì tuyến xe bạn cần truy vấn sẽ xuất hiện trên bản đồ với đường đi rõ ràng được mô tả bằng màu đỏ đậm, kèm thêm thông tin liên quan đến tuyến xe gồm: Số tuyến xe,lộ trình đi của xe bus, giá vé, giờ kết thúc, giờ xuất phát.

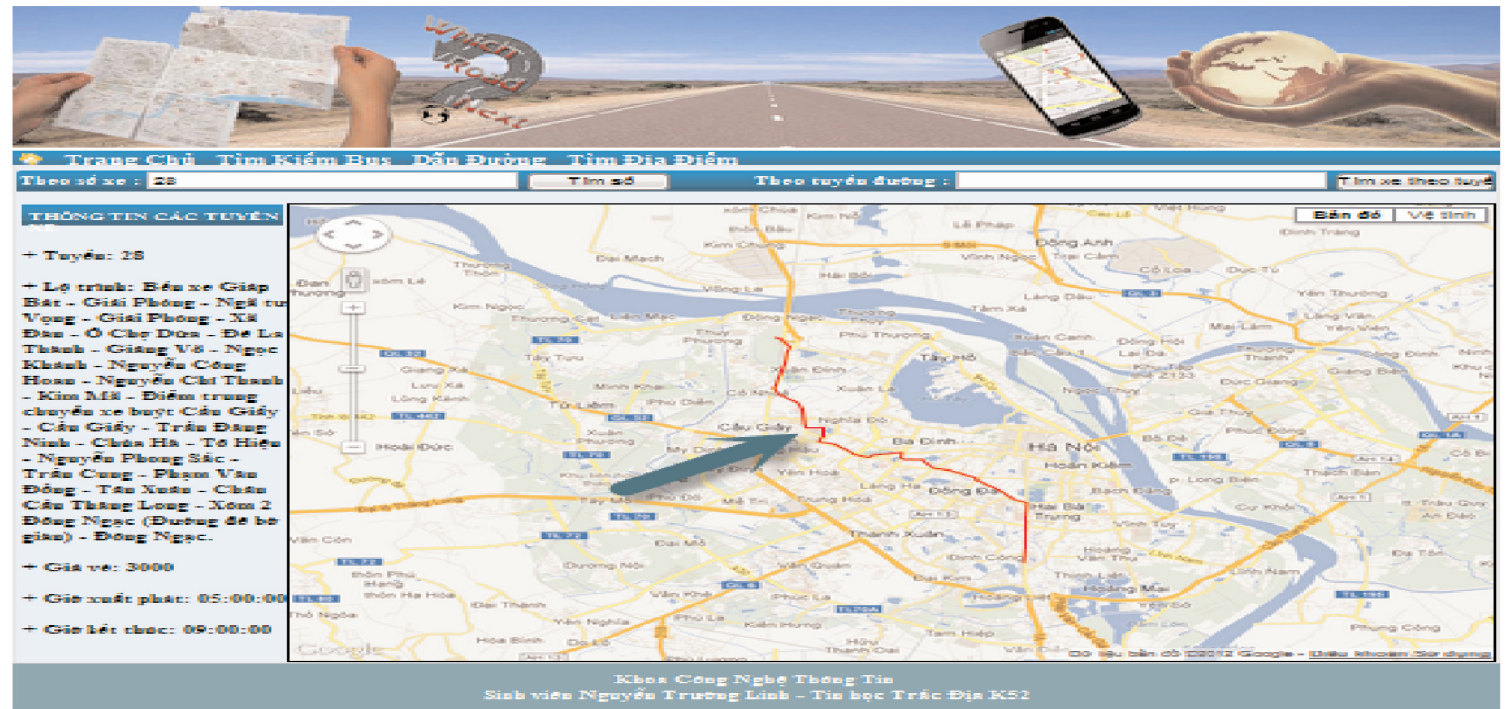

Hình 3: Tìm xe Bus theo số xe

c. Thực hiện chức năng tìm kiếm xe bus theo tuyến đường xe

Chức năng này trợ giúp người dùng tìm xem trên một tuyến đường cụ thể (ví dụ Đường Phạm Văn Đồng) có những tuyến xe nào đi qua.

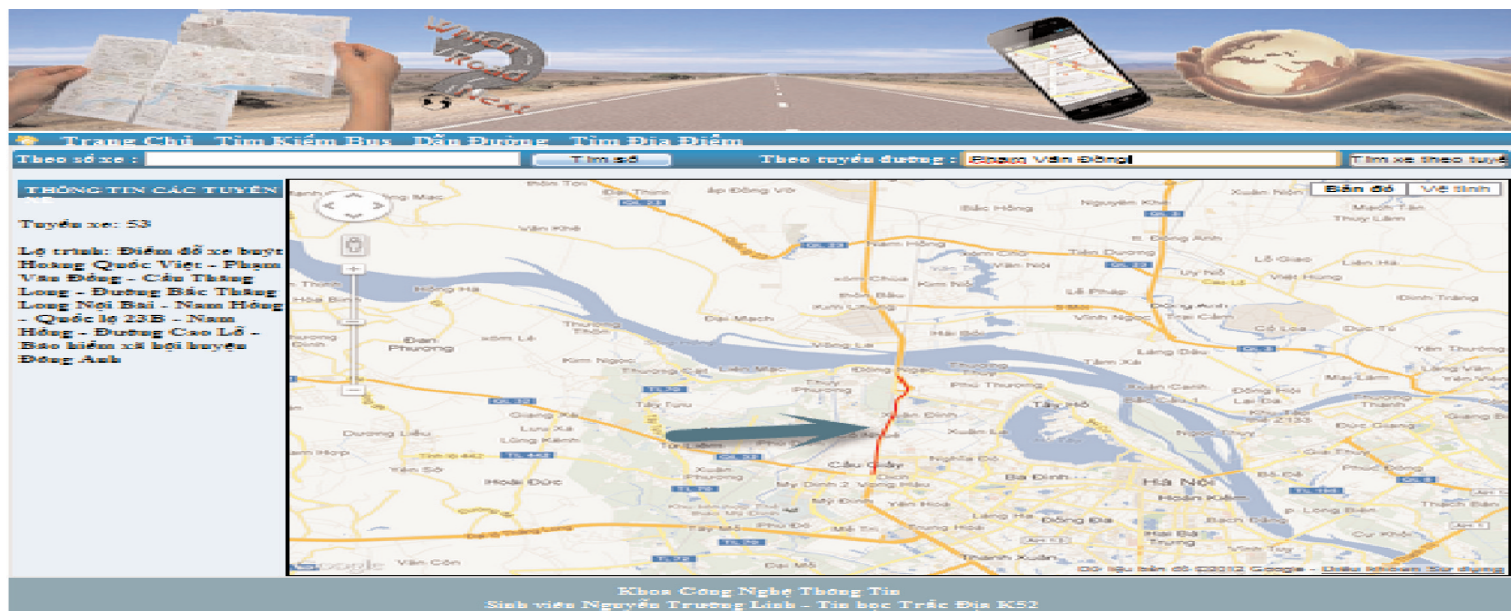

Hình 4: Tìm kiếm xe Bus theo tuyến đường 
d. Thực hiện chức năng tìm kiếm địa điểm trên bản đồ

Sau khi gõ địa chỉ cần tìm kiếm vào ô trên rồi click vào nút tìm kiếm thì địa điểm bạn cần tìm sẽ được đánh dấu ngay trên bản đồ và bản đồ sẽ được chuyển về vị trí điểm đánh dấu để bạn dễ dàng quan sát. Nếu muốn quan sát kỹ hơn thì chỉ việc zoom bản đồ tới địa điểm đó. Còn nếu không tìm thấy thì một hộp thoại thông báo lỗi sẽ hiện ra.Để làm được điều này thì chúng ta sử dụng hàm codeAddress

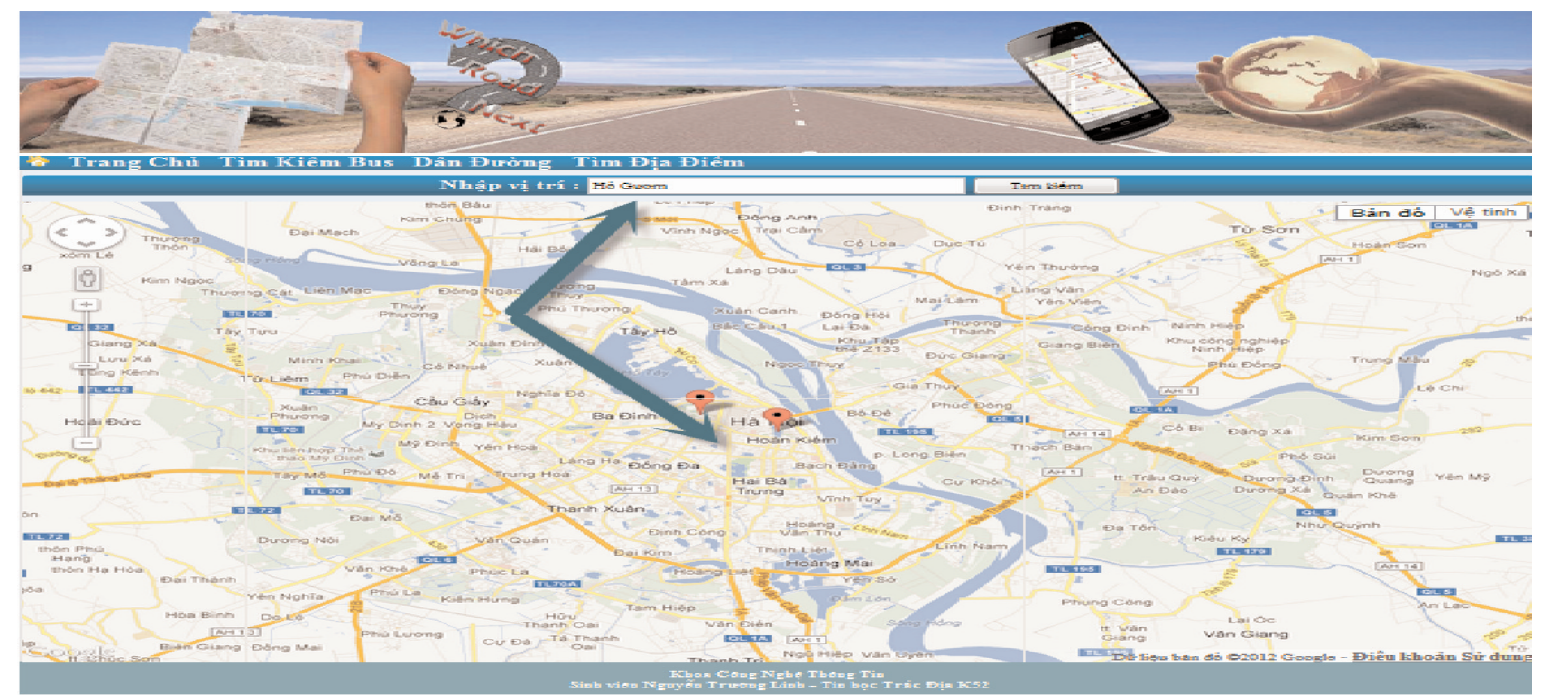

Hình 5: Tìm kiếm địa điểm trên bản đồ

Dưới đây là hàm rút gọn mô tả chức năng tìm kiếm được sử dụng trong website.

$<$ html $><$ head $>$

$<$ title $>$ Ha Noi Map </title $>$

<script src="http://maps.google.com/maps?file=api\&amp;v=2\&amp;key= ABQIAAAAuPsJpk3MBtDpJ4G8cqBnjRRaGTYH6UMI8mADNaOYKuWNNa8VNxQCzVBX Tx2DYyXGsTOxpWhvIG7Djw" type="text/javascript"></script>

$<$ script type="text/javascript">

var geocoder;

var map;

var infowindow = new google.maps. InfoWindow ();

var marker;

function codeAddress () \{

var address = document.getElementByld ("address").value;

if (geocoder) \{

geocoder.geocode (\{'address': address\}, function (results, status) \{

if (status $==$ google. maps.GeocoderStatus. OK) \{ 
map.setCenter (results[0].geometry.location);

var marker $=$ new google. maps. Marker $(\{$

map: map,

position: results[0].geometry.location

\});

\} else \{

alert ("Không tìm thấy địa điểm bạn cần tìm. Vui lòng xem lại bạn đánh đúng tên địa điểm muốn tìm chưa");

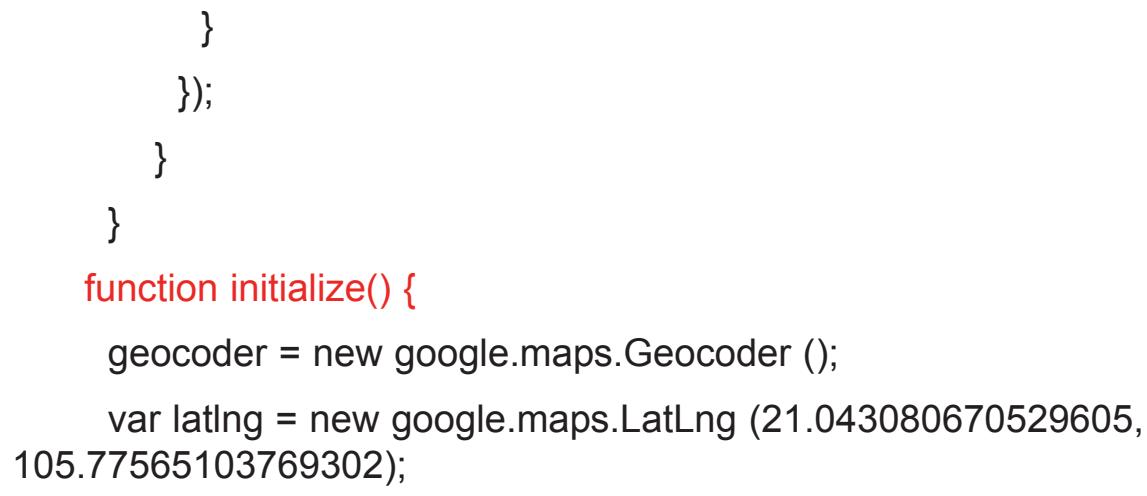

e. Thực hiện chức năng dẫn đường từ hai địa điểm biết trước

Chức năng cho phép tìm quãng đường ngắn nhất giữa hai địa điểm cho trước và có hướng dẫn cụ thể để đi từ địa điểm này đến địa điểm kia. Sau khi gõ hai địa chỉ cần dẫn đường vào ô trên rồi click vào nút tìm kiếm thì bản đồ dẫn đường sẽ hiển thị cho bạn xem, 
đường đi là đường được đánh dấu màu xanh đậm trên bản đồ. Nếu muốn quan sát kỹ hơn thì chỉ việc zoom bản đồ tới địa điểm đó. Bên cạnh đó là một bảng chỉ dẫn đường đi chi tiêt chỉ rõ khoảng cách 2 điểm là bao nhiêu, đi theo đường nào, quãng đường đi trên đường này bao nhiêu, và tới chỗ nào thì rẽ... sẽ được hướng dẫn chi tiết cho người dùng.

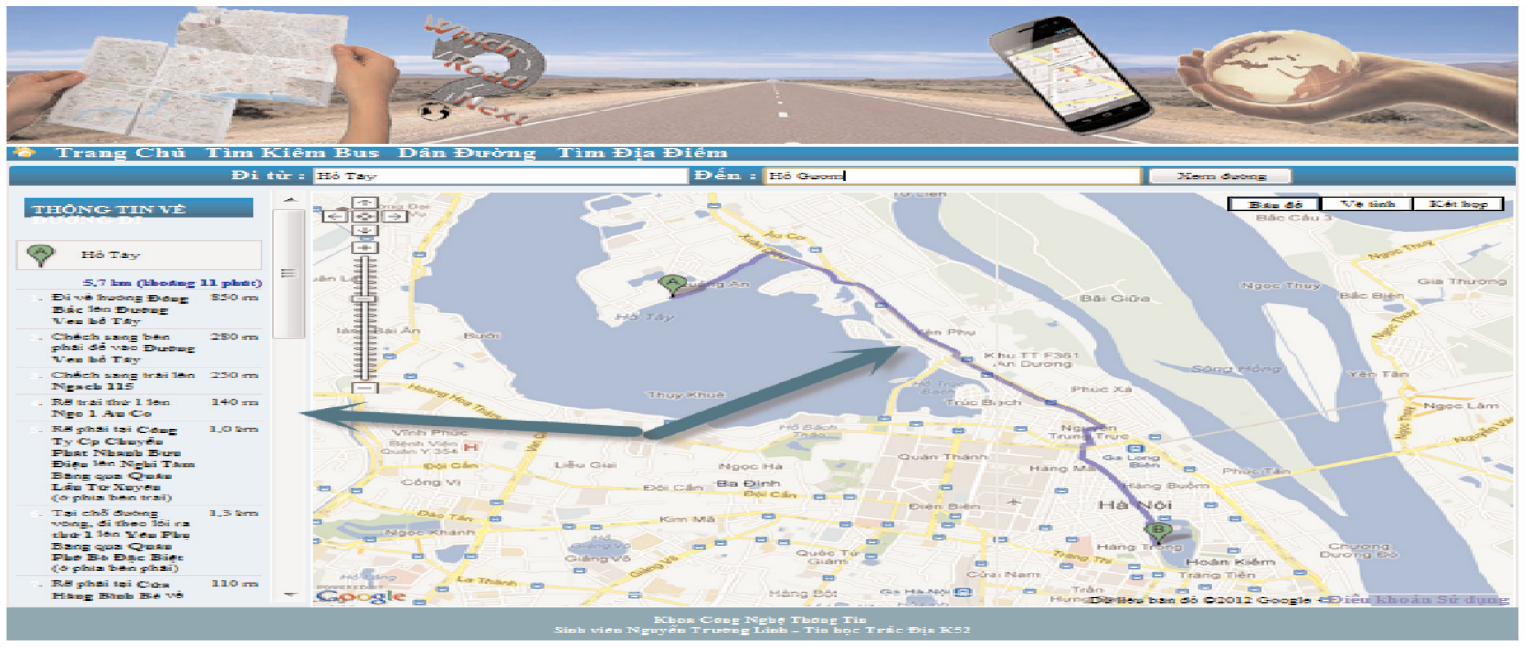

Hình 6: Chức năng dẫn đường

Dưới đây là hàm rút gọn mô tả chức năng dẫn đường được sử dụng trong website.

$<$ html>

$<$ head $>$

$<$ title $>$ Ha Noi Map </title>

<script src="http://maps.google.com/maps?file=api\&amp;v=2\&amp;key=

ABQIAAAAuPsJpk3MBtDpJ4G8cqBnjRRaGTYH6UMI8mADNaOYKuWNNa8VNxQCzVBX Tx2DYyXGsTOxpWhvIG7Djw" type="text/javascript"></script>

<script type="text/javascript">

var map;

var locale="vi";

var gdir;

var geocoder $=$ null;

var addressMarker;

function initialize() \{

if (GBrowserlsCompatible()) \{

map = new GMap2(document.getElementByld(“map_canvas”));

map.addControl (new GLargeMapControl ());

map.addControl (new GMapTypeControl ()); 
gdir = new GDirections(map, document.getElementByld("directions"));

GEvent.addListener (gdir, "load”, onGDirectionsLoad);

GEvent.addListener (gdir, "error", handleErrors);

SetDirections (“<?php echo \$_POST['from_way'];?>”, “<?php echo \$_POST['to_way'];?>”, "vi”);

\}

\}

function setDirections (fromAddress, toAddress, locale) \{

fromadd=fromAddress;

toadd=toAddress;

var hn=", hà nộ";

fromadd $=$ fromadd.concat $(\mathrm{hn})$;

toadd $=$ toadd.concat $(\mathrm{hn})$

gdir.load ("from: " + fromadd + " to: " + toadd, \{“locale": locale\});

\}

function handleErrors ()\{

if (gdir.getStatus().code $==$ G_GEO_UNKNOWN_ADDRESS)

alert ("Không tìm thấy địa chỉ mà bạn muốn tìm đường đi. Vui lòng xem lại tên đường và Tìm kiêm lại “ );

else if (gdir.getStatus ().code $==$ G_GEO_SERVER_ERROR)

alert("Một mã địa lý hoặc yêu cầu hướng dẫn có thể không được xử lý thành công, hoặc địa điểm cần tìm là không xác định được");

else if (gdir.getStatus ().code $==$ G_GEO_MISSING_QUERY)

alert(“Lỗi truy vấn” + gdir.getStatus ().code);

else if (gdir.getStatus ().code $==$ G_GEO_BAD_KEY)

alert ("Từ khóa cho là không hợp lệ hoặc không phù hợp ");

else if (gdir.getStatus ().code $==G_{-}$GEO_BAD_REQUEST)

alert ("Địa chỉ yêu cầu không thể phân tích thành công.");

else alert (“Không xác định được lỗi.");

\} 


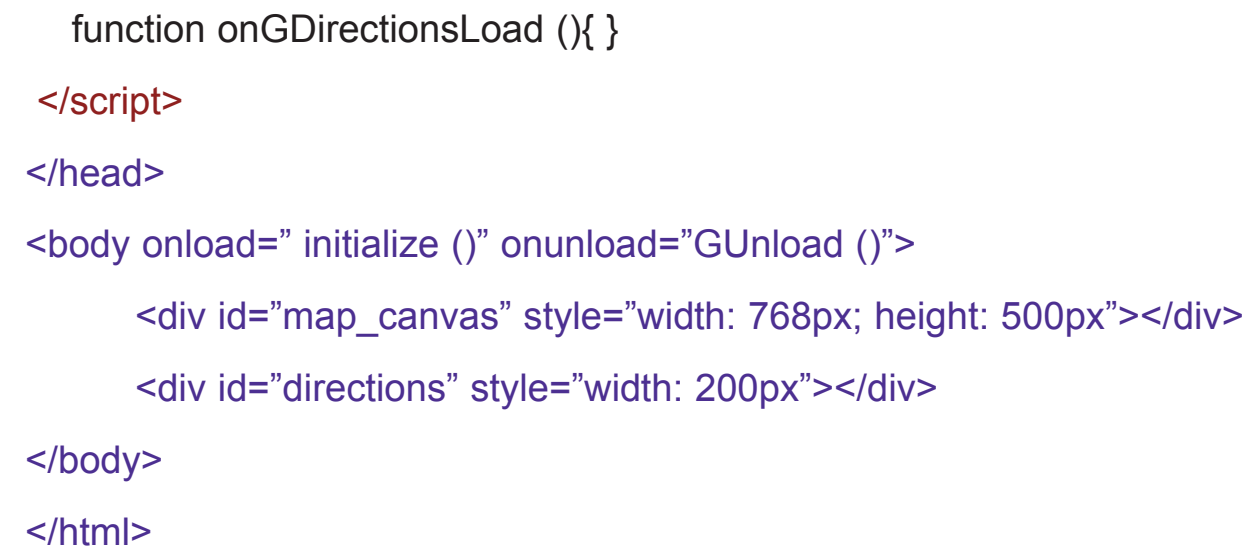

\section{Kết luận}

Website tra cứu thông tin địa lý và thông tin xe Bus giao diện trực quan, tiện dụng, tương tác tốt. Cho phép người dùng tra cứu, xem thông tin các tuyến xe một cách nhanh chóng.

Hướng nghiên cứu tiếp theo là bổ sung thêm các thông tin về các phương tiện giao thông khác như taxi, các tuyến xe liên tỉnh... $\mathrm{O}$

\section{Tài liệu tham khảo}

[1]. Tổng công ty vận tải Hà nội: www.transerco./d efault.aspx?pageid=253

[2]. Trang chủ Google Developer: https://developers.google.com/maps/

[3]. Trang Google Maps API Tutorial: http://econym.org.uk/gmap/

[4]. Các tool Google maps online: http://www.birdtheme.org/useful/googletool.html. $\bigcirc$

\section{Summary}

\section{Developing Website of Hanoi's bus}

\section{Assoc. Prof. Dr. Nguyen Truong Xuan}

Eng. Nguyen Truong Linh

Hanoi University of Mining and Geology

Eng. Mai Van Sy

Haiphong construction design and consultant joint stock company

In this paper we introduce the web site of Hanoi's Bus. This web site is developed by using JavaScript and Google Map APIs. User can access this web site by using PC, Laptops or smart phones to search information about all Hanoi's Bus. $\bigcirc$

Ngày nhận bài: 26/7/2013. 